\title{
Dual-Cluster Heads Clustering Routing Algorithm Based On Particle Swarm Optimization
}

\author{
Changjiang Jiang ${ }^{a}$, Hancheng Zhang ${ }^{b}$ and Yuwei Zhou, Lie Lei, Yun Ren \\ School of Chongqing University Of Posts And Telecommunications, Chongqing 400065, China; \\ accqjcj@qq.com, bcqupthczhang@163.com
}

\begin{abstract}
Keywords: Wireless Sensor Network(WSN) optimal number of cluster heads Particle Swarm Optimization(PSO) fitness function dual-cluster heads
\end{abstract}

\begin{abstract}
In order to solve the problem of the cluster head heavy energy consumption and energy utilization rate are insufficient in the clustering of wireless sensor network, proposed a dual cluster selecting based on particle swarm algorithm. Adopted the LEACH algorithm to cluster the area. Introduced PSO algorithm in each area, the cluster head is responsible for dataing collection and fusion, vice cluster head is responsible for forwarding data. Cluster head fitness function established by node energy and distances, the vice cluster head fitness established by three factors which were node energy, distance the vice cluster head of sink node, distance between the main cluster head to vice cluster head. The data transmission adopted one hop in cluster, multiple hops among the cluster. Compared with the LEACH protocol and the PSO-DH, the simulation results demonstrate that the method makes the network life extends $68 \%$ and $19 \%$, makes die nodes evenly distribution.
\end{abstract}

\section{Introduction}

Wireless Sensor Network WSN (Wireless Sensor Network) applied widely around us. Especially the area which people cannot reach, wireless sensor network can monitor these areas. The wireless sensor network will collect the data and deal these data then translate to the observers ${ }^{[1]}$. But supply the energy of the wireless sensor network's nodes is very difficult. So, how to efficient using the energy becomes one of the hot spot of current research in wireless sensor network (WSN).

The early traditional clustering routing protocols, such as $\mathrm{LEACH}^{[2]}$, EEUC ${ }^{[3]}$ and so on, these protocols have a one common problem which is consuming energy too fast of the cluster head. Aimed at these problem in the literature ${ }^{[4]}$, the new protocol clustering algorithm have introduced the particle swarm algorithm to optimize the selection of cluster heads, but in this protocol also adopt a single cluster, the problem of excessive consumption energy of the cluster head is still there. In the literature ${ }^{[5]}$, introducing the improved PSO algorithm adjusted the fitness function value and optimize the cluster head selection. Obviously, the algorithm can better balanced energy consumption within clusters. In addition, there are some routing protocols based on dual cluster head, such as EEIABR ${ }^{[6]}$, PSO - DH ${ }^{[7]}$.

\section{The Method}

2.1. PSO algorithm. Particle swarm optimization (PSO) algorithm is put forward by Eberhart etc $^{[8]}$. PSO algorithm will initialize a group of random particles, particles in search space through multiple iterations finally find the optimal solution. Suppose in D dimension position is expressed as vectors $x_{i}=\left(x_{i 1}, x_{i 2}, \cdots, x_{i d}\right)$, the number $\mathrm{i}$ of the particle has experienced the best location (best fitness) $x_{i d}=x_{i d}+v_{i d}$ is the vector $P_{i}=\left(p_{g 1}, p_{g 2}, \cdots, p_{g d}\right)$. Each particle speed vector is $V_{i}=\left(v_{i 1}, v_{i 1}, \cdots v_{i d}\right), i=1,2, \cdots, m$. The whole group, particles experienced all the best position vector is $P_{g}=\left(p_{g 1}, p_{g 2}, \cdots, p_{g d}\right)$. Each generation of particles according to the formula (1) and (2) update speed:

$$
v_{i d}=w v_{i d}+c_{1} r_{1}\left(p_{i d}-x_{i d}\right)+c_{2} r_{2}\left(p_{g d}-x_{i d}\right)
$$




$$
x_{i d}=x_{i d}+v_{i d}
$$

In the formula, $w$ is Inertia weight coefficient; $c_{1}$ and $c_{2}$ is Learning factor; $r_{1}$ and $r_{2}$ is a random number between $[0,1]$.

2.2. Cluster clustering routing algorithm based on PSO. This algorithm adopts the improved LEACH algorithm for clustering, Using PSO algorithm to optimize a main and vice cluster heads selection, data transmission using one jump in the cluster area, between clusters using multi-jump.

2.2.1 The optimal number of cluster heads. The number of cluster heads directly influences the whole network energy consumption. So how to calculate the reasonable number of optimal cluster heads is very important. Based on literature ${ }^{[9]}$, in the $\mathrm{M}^{*} \mathrm{M}$ monitoring areas that contains $\mathrm{N}$ nodes. Now we do not consider cluster heads and data forwarding. The optimal cluster Numbers is shown :

$$
k_{o p t}=\frac{\sqrt{N}}{\sqrt{2 \pi}} \sqrt{\frac{\boldsymbol{\varepsilon}_{f S}}{\varepsilon_{\text {amp }}}} \frac{M}{d_{t o-B s}^{2}}
$$

In the formula, $\varepsilon_{f s}$ is free space transmission mode power amplifier parameter. $\varepsilon_{\text {amp }}$ is the parameters of multipath transmission mode power amplifier.

Optimal cluster Numbers probability (probability of optimal cluster heads factor):

$$
k=\frac{k_{o p t}}{N}
$$

2.2.2 Divide the clustering area. In the initialization dividing cluster, Using the improved LEACH algorithm to select the cluster head, threshold formula is as follows:

$$
T(n)=\left\{\begin{array}{l}
\frac{K}{1-K\left(r * \bmod \frac{1}{K}\right)} \frac{E_{i}(r)}{E_{a v g}(r)}, n \in G \\
0, n \notin G
\end{array}\right.
$$

In formula (5), $\mathrm{K}$ is the optimal cluster heads probability, $\mathrm{r}$ is the number of cluster head election round, $\mathrm{G}$ is collection which respects the cluster head nodes of recent $1 / \mathrm{p}$ round. $E_{i}(r)$ is the residual energy of node $\mathrm{i}$ in the number $\mathrm{r}$ wheel. $E_{\text {avg }}(r)$ is the average residual energy of nodes in these area.

\subsection{Based on PSO algorithm to optimize dual cluster head selection}

2.3.1 PSO algorithm selection of fitness function. This article adopts the PSO algorithm is used for optimizing dual cluster head position. Because the fitness function plays an important role in the PSO algorithm. In this paper, according to different works, the cluster head is divided to main cluster-head and vice cluster-head. The main cluster head is responsible for collecting and merging the of node data. So, the nodes of the current energy is one of the important consideration of selecting main cluster-head. To sum up, the main cluster head to the fitness function is shown below:

$$
\begin{aligned}
& f=\alpha f_{1}+\beta f_{2} \\
& f_{1}=E(c) / \sum_{i=1}^{n} E(i) \\
& f_{2}=(n-1) / \sum_{i=1}^{n} d_{i t o C H}
\end{aligned}
$$

$f_{1}$ is the factors of cluster energy evaluation; $E(c)$ is the energy of the cluster heads. $\sum_{i=1}^{n} E(i)$ is the sum of all nodes energy among the cluster. $d_{i t o C H}$ is the node to the main cluster head's distance. $f_{2}$ is the factor of the main cluster-head compactness. Through debugging $\alpha$ and $\beta$, Choose the one makes the f maximum. And make the node into main cluster head. 
For the optimization of vice cluster-head, considering the vice cluster-head's main task is to transmit data to the base station. So, the high energy nodes and the short distance between to the main cluster-head and base station should be taken into account. Cluster fitness function is shown below:

$$
\begin{aligned}
& g=\gamma_{1} g_{1}+\gamma_{2} g_{2}+\gamma_{3} g_{3} \\
& g_{1}=E(c) / \sum_{i=1}^{n} E(i) \\
& g_{2}=d_{\text {VCHtoMVH }} / \sum_{i=1}^{n} d_{i t o M V H} \\
& g_{3}=d_{\text {VCHtoBS }} / \sum_{i=1}^{n} d_{i t o B S}
\end{aligned}
$$

$g_{1}$ is factor of vice cluster-head energy evaluation. $g_{2}$ is the Compactness factor of the vice cluster-head to the main cluster. $d_{V C H t o W V H}$ is the distance between vice cluster-head to the main cluster heads. $\sum_{i=1}^{n} d_{i t o \text { VVH }}$ is sum distance of the all ordinary nodes to the vice cluster-head. $g_{3}$ is the compactness of the vice cluster-head to the base station. $d_{V C H t o B S}$ is the distance between to the vice cluster-head and the base station. $\sum_{i=1}^{n} d_{i t o B S}$ is sum of all the ordinary node to the base station. Through debugging $\gamma_{1}, \gamma_{2}$ and $\gamma_{3}$, Choose the one makes the g maximum.

2.3.2 Dual cluster head selection process. According to the need of the algorithm in this paper, making the improvement on the traditional PSO algorithm, assuming that the network nodes in a plane coordinate, The position $x_{i d}$ and speed $v_{i d}$ of the network node i, decided by two directions of the $\mathrm{x}, \mathrm{y}$. So the formula (1) improvement as follows:

$$
\begin{aligned}
& V_{x i d}=w V_{x i d}+c_{1} r_{1}\left(p_{x i d}-x_{x i d}\right)+c_{2} r_{2}\left(p_{x g d}-x_{x i d}\right) \\
& V_{y i d}=w V_{y i d}+c_{1} r_{1}\left(p_{y i d}-x_{y i d}\right)+c_{2} r_{2}\left(p_{y g d}-x_{y i d}\right)
\end{aligned}
$$

formula (2) is improvement as:

$$
\begin{aligned}
& x_{x i d}=x_{x i d}+v_{x i d} \\
& y_{y i d}=y_{y i d}+v_{y i d}
\end{aligned}
$$

Steps: (1) Initialize particle swarm, within the cluster random initialization of the particle's position $x_{x i d}$ and $y_{y i d}$, speed $v_{x i d}$ and $v_{y i d}$ position for proofreading. (2) According to the formula (6) - (8) to calculate the fitness value. (3) Make individual extreme value is equal to the current position and find out all the particles of the appropriate value of the largest particle position as the global extremum, According to the formula (13) - (16) updates the node's position and velocity of each particle. (4) Through the formula (6) - (8) calculates value of the fitness function, the purpose is to updating all particles moderate values. (5) Repeat steps (3) (4), and ultimately to reach the largest number of iterations, choose the finally main cluster head. (6) According to fitness function (9), repeat the above operation, choose the finally vice cluster-head.

2.3.3 Data transmission. In data transmission phase, ordinary node sends the data to the main cluster-head in a one hop, vice cluster-head use multi-hops until the data reaches the node. Now the vice cluster-head data transmission process is divided into two steps. The first step, each vice cluster-head nodes first according to formula (17) calculates the function, then calculates their own distance between the base station $d_{1}$ (one hop threshold value is $d_{0}=\sqrt{E_{f s} / E_{m p}}$ ). if $d_{1}<d_{0}$ vice cluster-head adopt one hop data transmission mode; Otherwise, take multi-hops transfer mode. The second step, for each multi-hops vice cluster-head, they have to send broadcast its ID and their 
function values. Find the largest function values of the vice cluster-head nodes as the next hop node. Then next-hop repeat step 1 and 2 until transfer the data to the base station.

Selection of the next-hop function formula is as follows:

$$
N_{\text {next }}=\varepsilon \frac{E_{r}}{E_{i}}+(1-\varepsilon) \frac{d_{\max }-d_{i}}{d_{i}-d_{\min }}
$$

$\varepsilon$ is Weight coefficient; $E_{r}$ is the vice cluster-head surplus energy; $E_{i}$ is cluster heads initial energy; $d_{\max }$ is the longest distant in the network between nodes to base station, $d_{i}$ is distance between of node i to base station; $d_{\min }$ is the shortest distance between nodes to base station 。

\section{The results of simulation and analysis}

3.1 simulation environment. This article uses the MATLAB simulation platform, the simulation assume the 200 nodes randomly deployed in the range of $200 \mathrm{~m} * 200 \mathrm{~m}$. Simulation environment parameter Settings, such as table 1.

Table 1 The simulation environment of the parameter settings

\begin{tabular}{|c|c|c|c|}
\hline $\begin{array}{l}\text { The parameter } \\
\text { name }\end{array}$ & values & $\begin{array}{l}\text { The parameter } \\
\text { name }\end{array}$ & values \\
\hline $\begin{array}{c}\text { Network coverage } \\
\text { area }\end{array}$ & $(200,200) \mathrm{m}$ & $C_{1}$ & 2 \\
\hline $\begin{array}{l}\text { Base station } \\
\text { location }\end{array}$ & $(100,425) \mathrm{m}$ & $C_{2}$ & 2 \\
\hline Node Numbers & 200 & $W$ & 0.9 \\
\hline $\begin{array}{l}\text { Nodes initial } \\
\text { energy }\end{array}$ & $0.1 \mathrm{~J}$ & $\alpha$ & 0.5 \\
\hline Packet size & 2000bits & $\beta$ & 0.5 \\
\hline $\begin{array}{c}\text { Control package's } \\
\text { size }\end{array}$ & 100bits & $\gamma_{1}$ & 0.2 \\
\hline$E_{\text {elec }}$ & $50 \mathrm{~nJ} / \mathrm{b}$ & $\gamma_{2}$ & 0.3 \\
\hline$E_{\text {amp }}$ & $0.0013 \mathrm{pJ} / \mathrm{bit} / \mathrm{m}^{4}$ & $\gamma_{3}$ & 0.5 \\
\hline$E_{f s}$ & $10 \mathrm{~nJ} / \mathrm{bit} / \mathrm{m}^{2}$ & Rmax & 350 \\
\hline$\varepsilon$ & 0.4 & & \\
\hline
\end{tabular}

Figure 1. The area is $200 \mathrm{~m} * 200 \mathrm{~m}$. The three kinds of monitoring area of cluster distribution protocol. Distribution of cluster head of LEACH protocol (the "red +" on behalf of the cluster head) it can be seen that distribution of cluster heads is not reasonable. Distribution of cluster heads from PSO-DH protocol (red "+" on behalf of the cluster head, cluster, "red box" on behalf of the vice cluster head) can be seen in the relatively uniform, but the main and vice cluster head position is not well optimized. This article algorithm (red "+" on behalf of the cluster head, cluster "red box" on behalf of the vice cluster-head). Obviously cluster head distribution more uniform and the position of main and vice cluster heads are well optimized, effectively reduce the energy consumption of data transmission. 


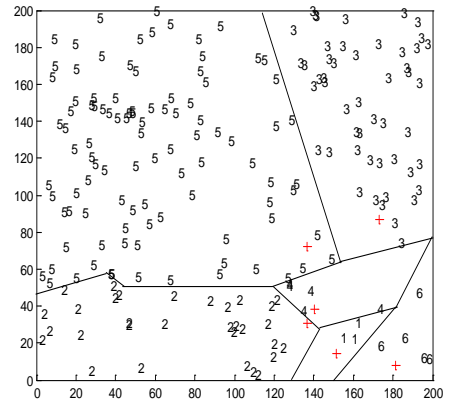

(a) LEACH protocol cluster distribution

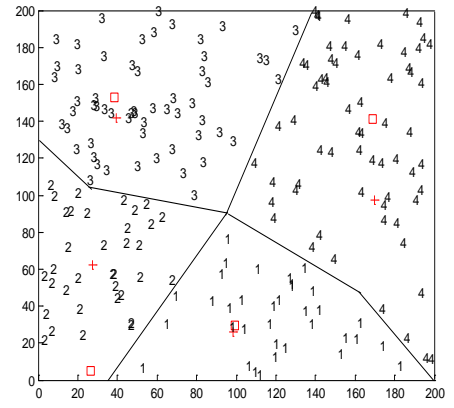

(b) PSO-DH protocol cluster distribution

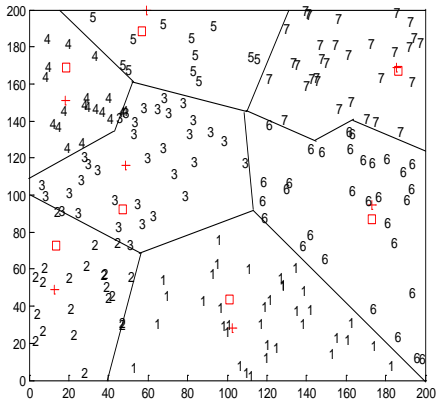

(c) This article Cluster distribution

Fig.1 Cluster head distribution

Figure 2. The area is $200 \mathrm{~m} * 200 \mathrm{~m}$, dead nodes distribution (" o "on behalf of the dead nodes, ". " on behalf of the survival nodes). Comparing three kinds of algorithms dead nodes distribution, when the dead node reached the $60 \%$ of the total node number in the area. LEACH protocol is concentrated hotspot problems are more obviously. PSO-DH dead nodes also have hotspot problem. This article algorithm can effectively relieve the hotspot problem.

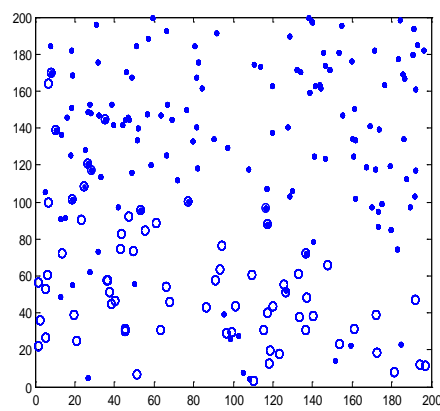

(a) LEACH

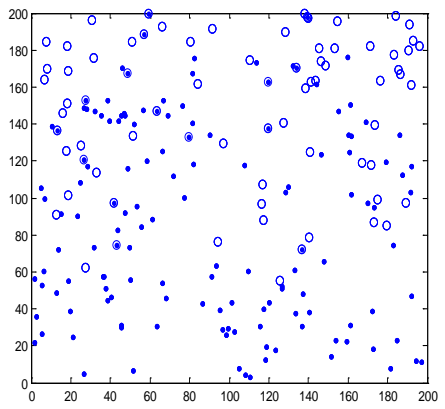

(b) PSO-DH

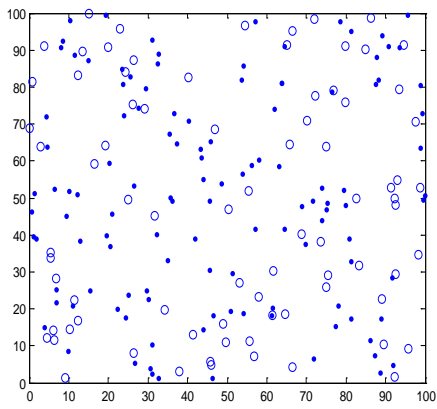

(c) This article protocol

Fig.2 Death node distribution

Figure 3. The network life cycle comparison. In the paper defines the first nodes death time is the network life cycle, clearly see from the picture in this paper protocol's network life cycle is the longest. PSO-DH is medium, the death node firstly occur is the LEACH protocol, The PSO - DH algorithm compared with LEACH algorithm, prolong the network life cycle by $49 \%$, and the article proposed algorithm is compared with the LEACH algorithm prolong the network life cycle by $68 \%$.

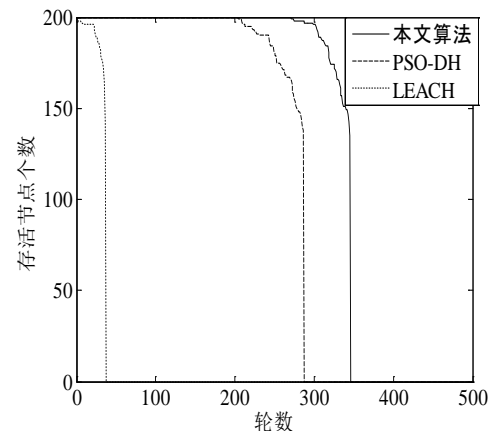

Fig.3 Life cycle comparison

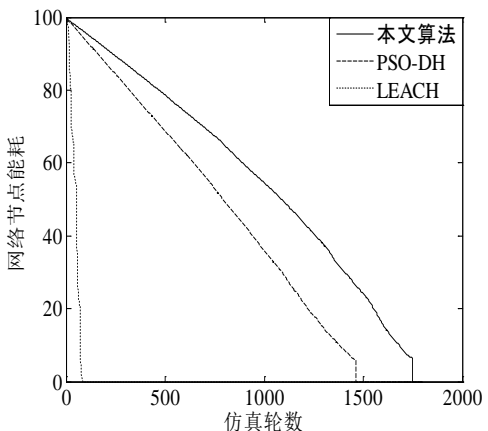

Fig.4 Total energy consumption of the network

Figure 4. The 3 kinds of protocol of the energy consumption in the network along with the change of the simulation cycle curve. In the simulation node set initial energy of 0.5 J. Smaller slope reflect the slower energy consumption and long life cycle. It can be seen from the diagram in this paper, the 
algorithm of total energy consumption is the most slow, compared with other two contrast protocol has obvious improved, so the use of the improved PSO algorithm to optimize dual cluster head selection can reduce the energy consumption.

\section{Summary}

Energy consumption is a hot issue in wireless sensor networks. Considering many factors of the clustering routing protocol, based on the optimal number of cluster heads and the nodes of the current energy factors make a cluster head election more reasonable. At the end of the clustered, using the improved PSO algorithm in clustering area, find out the optimal location of dual cluster heads. The last step is the data transmission to the one hop in the cluster and multi-hop between the clusters.

\section{References}

[1] SUN Limin, LI JianZhong, CHEN Yu . et al. Wireless Senor Networks [M]. Beijing: Tsinghua University Press, 2005:4-9.

[2] Heinzelman W R, Chandrakasan A, Balakrishnan H. Energy Efficient Communication Protocol for Wireless Mircrosensor Networks. In: Proc. of the 33rd Hawaii International Conference on System Sciences, 2000:4-7.

[3] LI Chengfa, CHEN Guihai, YE Mao. et al. An uneven cluster based routing protocol for wireless sensor networks $[\mathrm{J}]$.

Journal of Computers, 2007,30 (1):27- 36.

[4] LIU Zhikun, LIU Zhong, LI Zhaoxu. A Cluster Protocol for Wireless Sensor Networks Based on Chaos-PSO Optimization.[J]. Journal of Sensors And Actuators, 2011,24(10): 1459-1463

[5] XIE Zhibin, YU Qian, SHEN Bin, LIU Qinghua, TIAN Yubo. A New Dual-Cluster Heads Clustering Routing Algorithm Based on PSO[J]; Journal of Sensors And Actuators; 2013, 26(08) : 1135-1139.

[6] TENG Zhijun, ZHANG Fan, SONG Minghui. Wireless sensor network energy balance ant colony routing algorithm[J]. Journal of Jilin University 2016,46(1),327-332.

[7] HAN Dongxue, ZHANG Ruihua, LIU Danhua. PSO-based Double Cluster-heads Clustering Algorithm for Wireless Senor Network[J]. Journal of Computer Engineering, 2010, 36(10) : 100-102. [8] Kennedy J, Eberhart R C. Particle Swarm Optimization[C] //Proceeding of IEEE International Conference on Neural Networks, Perth, Australia, 1995, 4 : 1942-1948.

[9] TANG Yi, LIANG Xiaoxi, Wu Jun. A Research of the Optimal Number of Cluster Heads of Wireless Sensor Networks[J]. Journal of communications techology,2007,6:30-32.

\section{Acknowledgments}

This work was financially supported by National Ministry of industry and the development of the Internet of things special fund projects (Financial enterprises in Chongqing, code :[2012]776 ) of China. The paper's title is "Dual-Cluster Heads Clustering Routing Algorithm Based On Particle Swarm Optimization", and the author of communication is ZHANG Hancheng. His email is : cqupthczhang@163.com 